Article

\title{
Fc Gamma Receptor IIb Expressed in Hepatocytes Promotes Lipid Accumulation and Gluconeogenesis
}

\author{
Ting Shu, Xiaomin Song, Xingxing Cui, Weipeng Ge, Ran Gao and Jing Wang * \\ State Key Laboratory of Medical Molecular Biology, Institute of Basic Medical Sciences, Chinese Academy of \\ Medical Sciences, Department of Pathophysiology, Peking Union Medical College, Beijing 100005, China; \\ 15901356250@126.com (T.S.); 18271392658@163.com (X.S.); cuixingxing90@163.com (X.C.); \\ 18840827758@163.com (W.G.); gaoran@ibms.pumc.edu.cn (R.G.) \\ * Correspondence: wangjing@ibms.pumc.edu.cn; Tel.: +86-010-69156477
}

Received: 10 August 2018; Accepted: 8 September 2018; Published: 13 September 2018

check for updates

\begin{abstract}
Non-alcoholic fatty liver disease (NAFLD) is characterized by ectopic lipid accumulation in the liver, usually combined with hepatic insulin resistance. Fc-gamma receptor-IIb (Fc $\gamma \mathrm{RIIb})$ and its ligand are reported to be associated with obesity and type 2 diabetes mellitus (T2DM). As knowledge about Fc $\gamma$ RIIb in the literature is mostly generated from studies on skeletal muscle tissue, the expression and function of Fc $\gamma$ RIIb in the liver and hepatocytes are largely unknown. In this study, we identified the expression of Fc $\gamma$ RIIb in primary cultured mouse hepatocytes: Fc $\gamma$ RIIb was upregulated in response to oleic acid (OA) in a dose dependent manner. Fc $\gamma$ RIlb knockdown using shRNA suppressed the lipid and triglyceride accumulation, and mRNA expression of ACC1, FASn, CD36, MTTP, and ApoB in OA-treated HepG2 cells. Fc $\gamma$ RIIb deficiency mice fed with high fat diet (HFD) had significantly lower liver weight and liver to body weight ratio, as well as less triglyceride accumulation in the livers. In glycometabolism, Fc $\gamma$ RIIb hindered insulin-induced phosphorylation of AKT and FOXO1, and in turn upregulated G6Pase and PEPCK mRNA expression, suggesting that Fc $\gamma$ RIIb promotes gluconeogenesis by suppressing the AKT/FOXO1/G6Pase/PEPCK pathway in hepatocytes. This study reveals a novel role for Fc $\gamma$ RIIb in regulating lipid metabolism and glycometabolism, and provides a new therapeutic target to improve NAFLD.
\end{abstract}

Keywords: Fc $\gamma$ RIIb; NAFLD; gluconeogenesis; insulin sensitivity

\section{Introduction}

Non-alcoholic fatty liver disease (NAFLD) is a progressive liver disease. Patients with NAFLD have ectopic lipid accumulation in liver, and are in a high risk of developing into non-alcoholic steatohepatitis (NASH), cirrhosis, hepatocellular carcinoma (HCC), and hepatic insulin resistance [1]. The prevalence of NAFLD reached $25-30 \%$ worldwide, and 30-40\% among them will get worse [2]. The main cause of NAFLD is the over nutrition and obesity. As a nexus of metabolic and hepatic disease [3], liver damage will cause hyperglycemia, hyperlipidemia, and type 2 diabetes mellitus (T2DM).

The liver is an organ in charge of the balance of energy storage and supply. Food intake evokes high concentration of insulin that activates insulin receptor. Activated insulin receptor promotes phosphorylation of AKT and its downstream factor, FOXO1 in hepatocyte [4]. As a transcription factor, phosphorylation of FOXO1 suppresses its translocation into nuclear where it is supposed to upregulate expression of G6Pase and PEPCK [5]. G6Pase and PEPCK are important enzymes for gluconeogenesis, which restrain hyperglycemia [3,4]. Liver also makes blood glucose stable in fasting state. However, under insulin resistance, accumulation of free fatty acid (FFA) in the liver, and high concentration of glucose in blood will occur [6]. Liver de novo lipogenesis and fatty acid esterification are the two 
main sources that promote lipid synthesis in hepatocyte $[2,3,7]$. Under normal physiological condition, glucose from blood is taken up into the hepatocyte to be synthesized into triglyceride by de novo lipogenesis related factors $S R E B P-1, A C C 1$ and FASn [3]. Serum FFA is transported into the hepatocyte by fatty acid transport proteins (such as CD36) and fatty acid binding proteins in the membrane. Intracellular fatty acid is esterified it into triglyceride [2]. Lipolysis also controls lipid accumulation in hepatocytes through fatty acid $\beta$-oxidation in mitochondria [8]. Nuclear receptor PPAR $\alpha$ controls every enzymatic step related gene within the fatty acid oxidation pathways, such as CPT1 $\alpha$ and MCAD [8]. Suppression of PPAR $\alpha$ makes fatty acid fail to import into the mitochondria, which results in low lipolysis and lipid accumulation in the hepatocyte [8].

Fc $\gamma$ RIIb, an Immuneglobulin G(IgG) receptor, is a tyrosine kinase receptor, located at the cell membrane. Fc $\gamma$ RIIb is the only inhibitory receptor of IgG [9]. Fc $\gamma$ RIIb transmits inhibitory signals through an immunoreceptor tyrosine-based inhibitory motif (ITIM) in its cytoplasmic region. Activation of Fc $\gamma$ RIlb triggers ITIM phosphorylation, and results in recruitment of phosphatases including a SH2 domain containing inositol polyphosphate $5^{\prime}$ phosphatase (SHIP) and a SH2-domain containing protein tyrosine phosphatase 1 [10]. Previous studies demonstrated that Fc $\gamma$ RIIb was associated with T2DM and obesity. Tanigaki reported that Fc $\gamma$ RIlb was expressed in skeletal muscle microvascular endothelium [11], and regulated endothelium insulin delivery and skeletal muscle glucose uptake using C Reactive Protein (CRP) transgenic mice and endothelial cell-specific Fc $\gamma$ RIIb knockout mice [12]. Tanigaki also reported that IgG from patients with T2DM and High Fat Diet-fed mice exhibited hyposialylation in its Fc glycan, which resulted in blunting endothelial insulin transcytosis [13]. However, it is unknown whether the Fc $\gamma$ RIIb ligand and its receptor axis existed in hepatocytes and if so, how it regulated hepatocyte function.

In our current study, we identified the expression of Fc $\gamma \mathrm{RIIb}$ in hepatocytes, and the novel role for Fc $\gamma$ RIIb in regulating hepatocytic lipid and glucose metabolism in vivo and in vitro. Our data suggest that Fc $\gamma$ RIIb promotes lipid accumulation and glyconeogenesis, which favor development of NAFLD and insulin resistance.

\section{Results}

\subsection{Fc $\gamma$ RIIb Expressed in Hepatocytes}

To assess if the Fc $\gamma \mathrm{RIIb}$ ligand and its receptor axis play a role in hepatocytes, we analyzed Fc $\gamma$ RIIb expression in isolated hepatocytes. Quantitative RT-PCR analysis showed that $F c \gamma R I I b$ mRNA was expressed in hepatocytes compared with the Fc $\gamma$ RIIb positive cell, RAW264.7, and that the mRNA level of $F c \gamma R I I b$ was markedly higher in primary cultured hepatocytes from wildtype $\left(\mathrm{Fc}_{\mathrm{C}} \mathrm{RIIb}{ }^{+/+}\right.$) mice than that in hepatocyte from Fc $\gamma$ RIIb knockout $\left(\mathrm{Fc}_{\mathrm{R}} \mathrm{RIIb}^{-/}{ }^{-}\right.$) mice (Figure 1A,B). Consistent with our qRT-PCR result, the level of Fc $\gamma$ RIIb protein was also markedly elevated in hepatocytes from Fc $\gamma \mathrm{RIIb}^{+/+}$mice detected in Western blot (Figure 1C). To examine the existence of Fc $\gamma \mathrm{RIIb}$ in intact hepatocytes, we performed immunofluorescence cell staining for Fc $\gamma$ RIIb in primary cultured hepatocytes. The results showed intensively positive staining with $\mathrm{F} c \gamma \mathrm{RIIb}^{+/+}$hepatocytes but not with Fc $\gamma \mathrm{RIIb}^{-/-}$hepatocytes (Figure 1D). These data suggest that Fc $\gamma \mathrm{RIIb}$ is expressed in hepatocytes. 

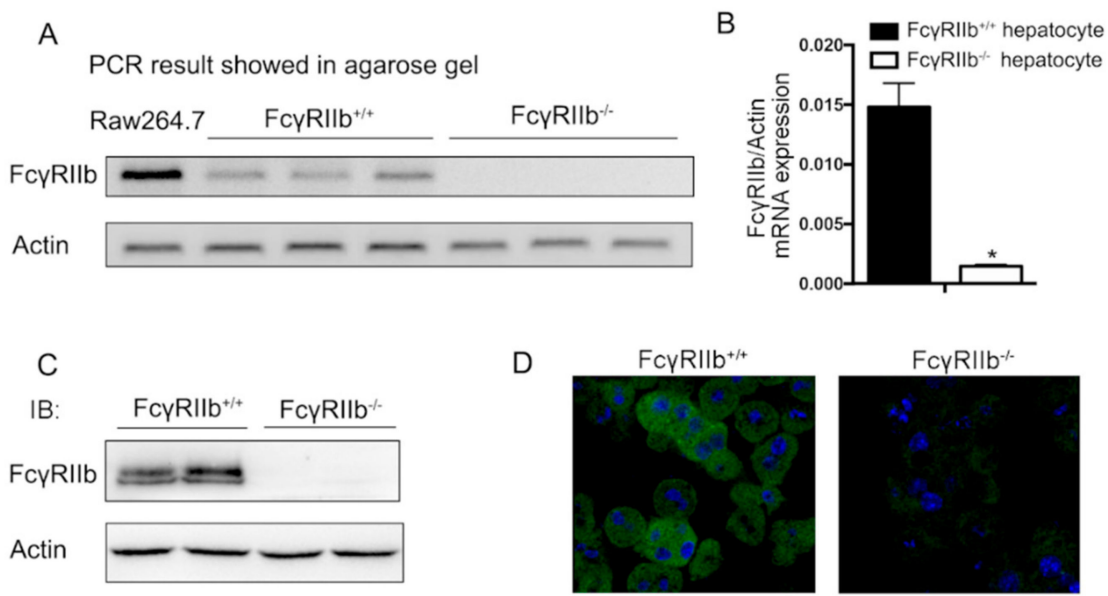

Figure 1. Expression of Fc $\gamma \mathrm{RIIb}$ in hepatocytes. Primary cultured mouse hepatocytes were isolated from Fc $\gamma \mathrm{RIIb}^{+/+}$and Fc $\gamma \mathrm{RIIb}^{-/-}$mice. (A) RNA was extracted and RT-PCR was performed using primers designed for amplification of Fc $\gamma$ RIIb. Macrophage celline RAW264.7 was used as a positive control. (B) Fc $\gamma$ RIIb/actin mRNA expression ratio, detected by qRT-PCR. ${ }^{*} p<0.05, n=3$ (C) Western blot for Fc $\gamma$ RIIb protein expression in primary cultured mouse hepatocyte. (D) ICC staining of Fc $\gamma$ RIlb in primary cultured mouse hepatocyte $(40 \times)$. Positive area is shown in green, DAPI is shown in blue.

\subsection{Increase of $F c \gamma R I I b$ Expression after Oleic Acid Treatment}

To explore the role of Fc $\gamma$ RIIb in regulating hepatocyte lipid metabolism, we examined the expression of $\mathrm{Fc} \gamma \mathrm{RIIb}$ expression in response to oleic acid (OA) in human hepatocarcinoma cell line HepG2, as OA was used to induce triglyceride accumulation [14]. After $24 \mathrm{~h}$ incubation with OA, the expression of $F c \gamma R I I b$ in HepG2 was detected by qRT-PCR and Western blot. The results showed that $F c \gamma R I I b$ expression in HepG2 was significantly upregulated in HepG2 treated with $0.4 \mathrm{mM} \mathrm{OA}$, and peaked with $0.8 \mathrm{mM}$ OA treatment at both mRNA and protein levels (Figure 2A,B). When treated with $0.4 \mathrm{mM} \mathrm{OA}, F c \gamma R I I b$ expression in HepG2 plateaued at $12 \mathrm{~h}$ after the OA treatment (Figure 2C,D). These results indicated that $F c \gamma R I I b$ was upregulated in response to OA treatment, and suggest that $F c \gamma R I I b$ may play a role in hepatocyte lipid metabolism.
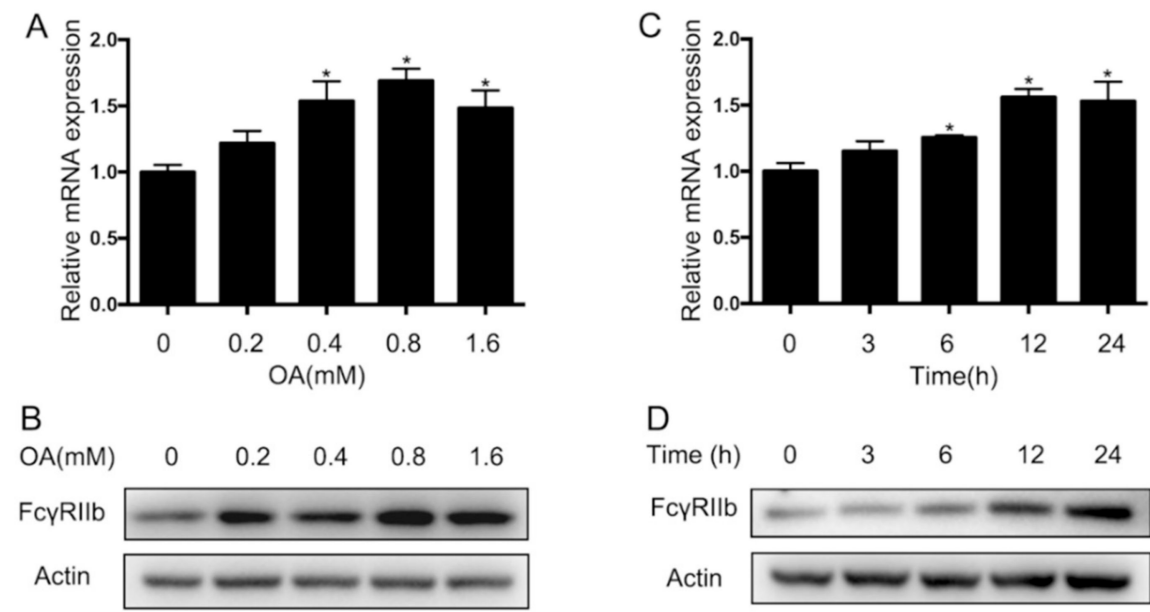

Figure 2. Fc $\gamma$ RIIb expression and regulation in HepG2. (A) qRT-PCR for mRNA expression of Fc $\gamma$ RIIb in HepG2 in response to the indicated doses of oleic acid (OA) for $24 \mathrm{~h}$. (B) Western blot analysis for Fc $\gamma$ RIIb expression under the same conditions as described in A. (C) qRT-PCR for mRNA expression of $F c \gamma R I I b$ in HepG2 cells in response to $0.4 \mathrm{mM}$ OA treatment for indicated periods. (D) Western blot analysis for detecting Fc $\gamma$ RIIb expression under the indicated conditions. The data shown are the means \pm SEM. ${ }^{*} p<0.05$. 


\subsection{Increase of Fc $\gamma$ RIIb Expression after Oleic Acid Treatment}

To verify the role of Fc $\gamma$ RIIb in hepatocyte lipid accumulation, HepG2 cells were transfected with lentivirus expressing Fc $\gamma$ RIIb shRNA or a control shRNA. With $48 \mathrm{~h}$ transfection, Fc $\gamma$ RIIb mRNA in the cells treated with Fc $\gamma$ RIIb shRNA-lentivirus was significantly decreased compared to the control shown in qRT-PCR (Figure 3A). Afterward, HepG2 cells transfected as described above were incubated with or without OA for $24 \mathrm{~h}$. Oil Red O staining showed no difference in lipid accumulation between the cells transfected with Fc $\gamma$ RIIb shRNA and control shRNA in basal level, but significantly fewer lipid droplets in the cells with Fc $\gamma$ RIIb knockdown compared with the control after OA treatment (Figure 3B,C). In accord with the Oil Red O staining, the total triglyceride assay showed significantly less triglyceride content in the cells with Fc $\gamma$ RIIb knockdown (Figure 3D). These results indicate that Fc $\gamma$ RIIb plays a role in mediating OA-induced lipid accumulation in hepatocyte.

To elucidate the targets of Fc $\gamma \mathrm{RIIb}$, qRT-PCR was performed to evaluate the putative genes involved in lipogenesis, fatty acid oxidation, and lipid transportation (Figure 3E) in control HepG2 cells with or without OA treatment, and knockdown Fc $\gamma \mathrm{RIIb}$ with OA treatment. The results showed that OA-treated HepG2 cells exhibited a marked increase in mRNA expression of the indicated lipogenesis genes (Figure 3E, the three groups in the left) and a mild increase in that of the lipolysis genes of interest (Figure 3E, the three groups in the middle), which favors lipid metabolism in the cells. However, knockdown of Fc $\gamma$ RIIb significantly resulted in reversions in the OA-induced upregulation of lipogenesis mRNAs compared to their controls ( $A C C 1$ and $F A S n)$, and lipid transport gene (CD36, $M T T P$ and $A p o B)$, but showed no inhibiting effect on expression of the indicated lipolysis genes (PPAR $\alpha$, $C P T 1 \alpha$ and $M C A D$ ). These results suggest that Fc $\gamma$ RIIb promotes lipid accumulation in hepatocytes through enhancing lipogenesis and lipid transportation.

\subsection{Fc $\gamma$ RIIb-Deficiency Attenuated HFD-Induced Hepatic Steatosis in Mice}

To examine the regulatory function of Fc $\gamma$ RIIb in hepatic lipid metabolism in vivo, the following experiments were performed in Fc $\gamma \mathrm{RIIb}$ knockout mice $\left(\mathrm{Fc} \gamma \mathrm{RIIb}^{-/-}\right)$and wild type mice $\left(\mathrm{Fc}_{\mathrm{R}} \mathrm{RIIb}^{+/+}\right)$. Wildtype mice were fed with the Normal Chow Diet (NCD) and High Fat Diet (HFD) to confirm if HFD induced hepatic steatosis in an animal model. HFD feeding induced significantly body weight gain (Figure 4A), liver weight gain (Figure 4B), and increased liver/body weight ratio (Figure 4C), compared with NCD. Gross morphology, pathology changes, and triglyceride content increase were shown in Figure 4D,F. This confirmed that HFD feeding induce fatty liver in mice. Under HFD feeding, $\mathrm{Fc}_{\mathrm{R}} \mathrm{RIIb}^{-/-}$mice exhibited no significant body weight gain (Figure 4A), but lower liver weight

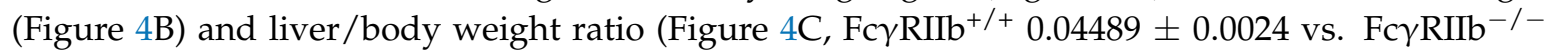
$0.0341 \pm 0.0026$ ). Gross morphology of Fc $\gamma \mathrm{RIIb}^{-/-}$mice showed less pale liver (Figure $4 \mathrm{D}$ ) which indicates less fat accumulation in liver, fewer lipid droplets, and vacuoles (Figure 4F), and lower triglyceride level (Figure 4E, Fc $\gamma \mathrm{RIIb}^{+/+} 3.465 \pm 0.171 \mathrm{vs.} \mathrm{Fc} \gamma \mathrm{RIIb}^{-/-} 1.555 \pm 0.529$ ). Moreover, serum alanine aminotransferase (ALT) and aspartate aminotransferase (AST), two biomarkers for liver injury, were measured. As shown in Figure 4G, serum ALT in Fc $\gamma \mathrm{RIIb}^{-/-}$mice were significantly lower than that in $\mathrm{Fc} \gamma \mathrm{RIIb}^{+/+}$mice, indicating less hepatocyte injury in Fc $\gamma \mathrm{RIIb}^{-/-}$mice. Gene mRNA expression was confirmed in the animal study (Figure $4 \mathrm{H}$ ). The upregulation of the genes of interest (SREBP-1, $A C C 1, F A S n$, and CD36) in HepG2 cells was verified in Fc $\gamma \mathrm{RIIb}^{+/+}$mice fed on NCD, and Fc $\gamma \mathrm{RIIb}^{+/+}$ or Fc $\gamma \mathrm{RIIb}^{-/-}$mice on HFD. HFD feeding resulted an upregulation of the genes of interest, which is supposed to promote lipogenesis and lipid transport. Knockout of Fc $\gamma$ RIIb significantly reversed this effect of HFD. These data support that our in vivo finding on the mechanism underlying the effect of Fc $\gamma$ RIIb on lipid accumulation reflects the physical event in vivo. 
A

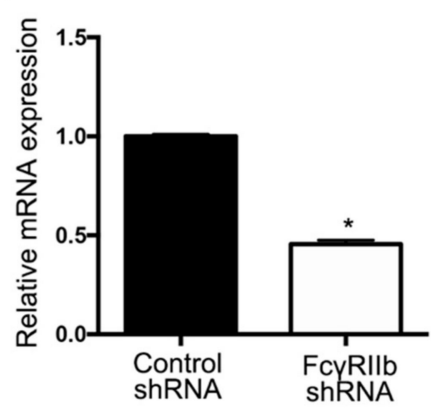

C

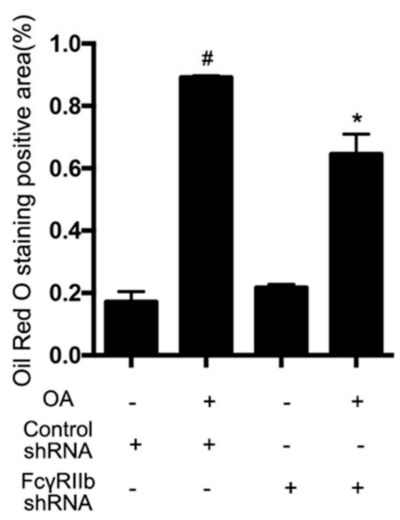

B
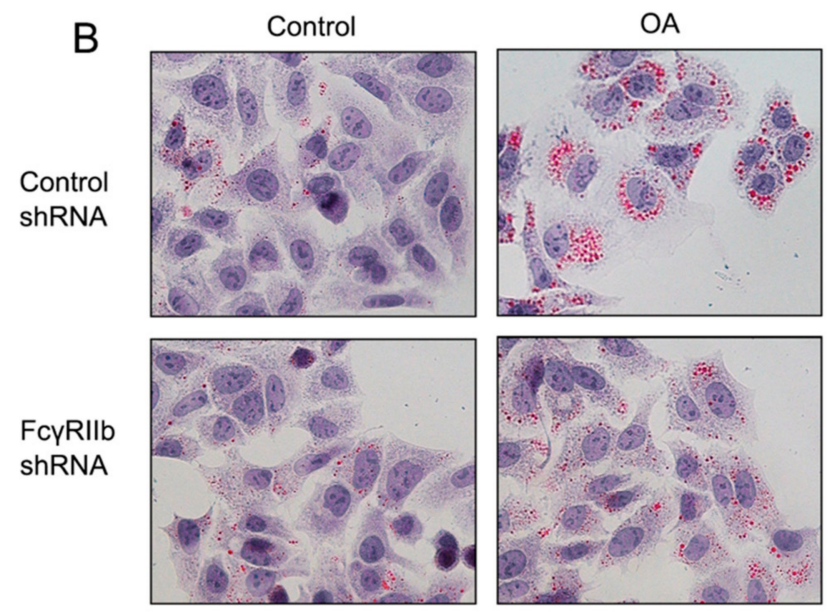

D

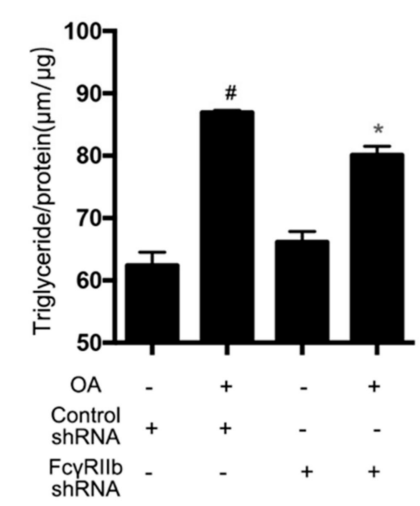

$\mathrm{E}$

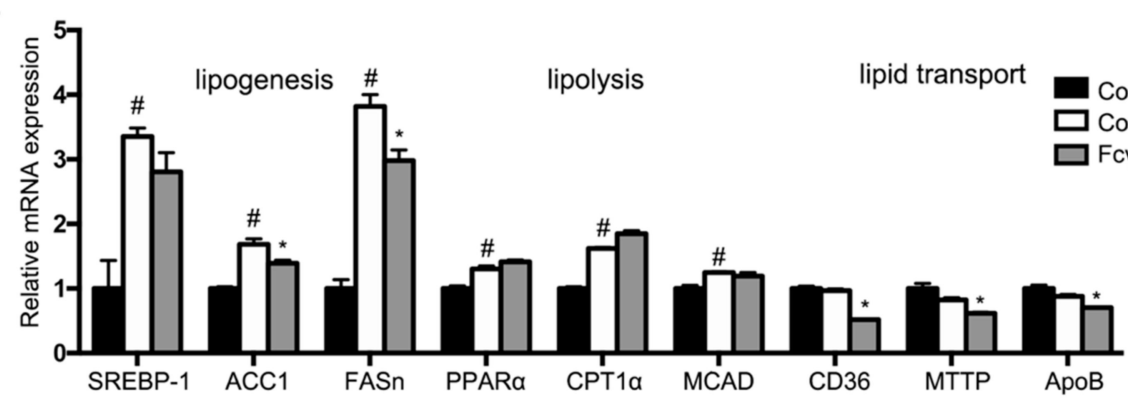

Figure 3. Effect of $F c \gamma R I I b$ knockdown on lipid accumulation in OA induced steatosis. (A) Relative mRNA expression of $F c \gamma$ RIIb in HepG2 $48 \mathrm{~h}$ after Fc $\gamma$ RIIb-shRNA and Control-shRNA administration. (B) Oil Red O staining $(40 \times)$ for lipid droplets in HepG2 after 24 h OA treatment. (C) Statistical analysis of Oil Red O staining positive areas. ${ }^{*} p<0.05$, between control-shRNA and Fc $\gamma$ RIIb-shRNA treatment under OA, ${ }^{*} p<0.05$, between basal and OA treament in control-shRNA. (D) Measurement of intracellular triglyceride content in HepG2. ${ }^{*} p<0.05$, between control-shRNA and Fc $\gamma$ RIIb-shRNA treatment under OA, ${ }^{\#} p<0.05$, between basal and OA treatment in control-shRNA transfected. (E) Relative mRNA expression of the genes involved in lipid synthesis, $\beta$-oxidation, and lipid transportation in control cell of basal level, knockdown, and control HepG2 after OA treatment. Gene expression was normalized to that of $\beta$-actin. The data shown are the means \pm SEM. ${ }^{*} p<0.05$, between control shRNA cell with and without OA treatment (bar 1 and bar 2); ${ }^{*} p<0.05$, between control and Fc $\gamma$ RIIb shRNA with OA treatment (bar 2 and bar 3). 
A

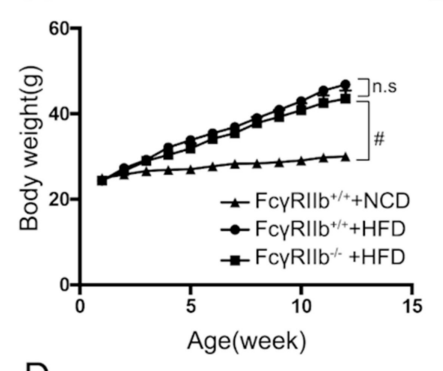

$\mathrm{D}$

$\mathrm{FCYRIIb}^{+/+}+\mathrm{NCD}$

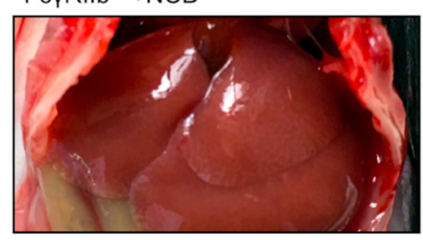

B

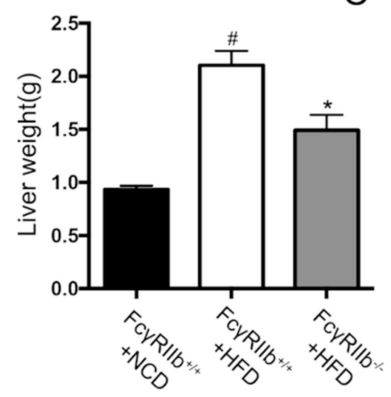

$\mathrm{FcYRIIb}^{+/+}+\mathrm{HFD}$

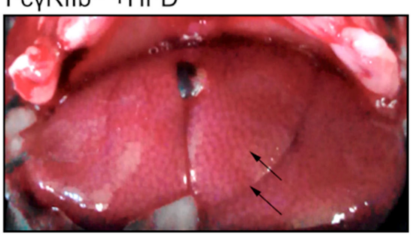

C

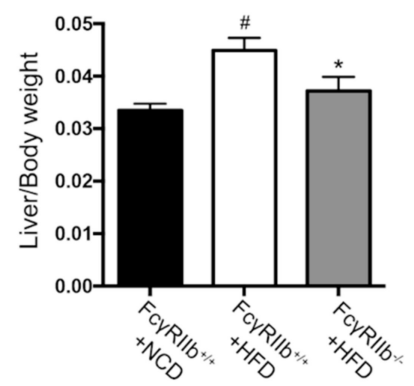

$\mathrm{FCYRIIb}^{-/+\mathrm{HFD}}$

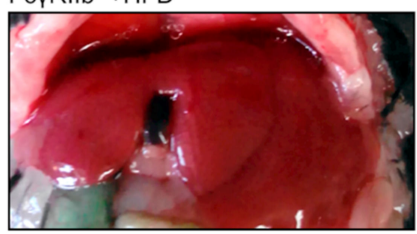

$\mathrm{E}$

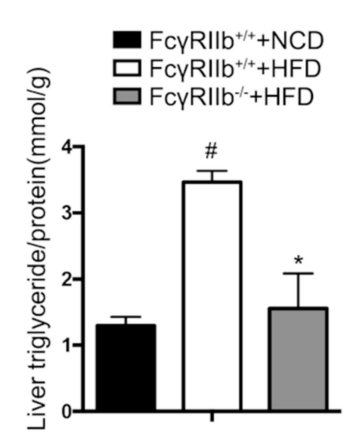

G

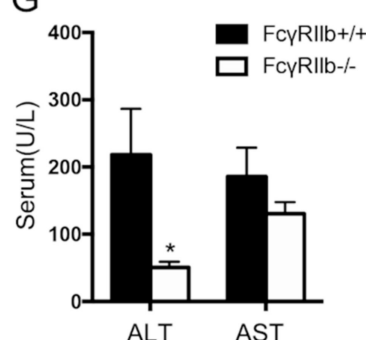

$\mathrm{F}$

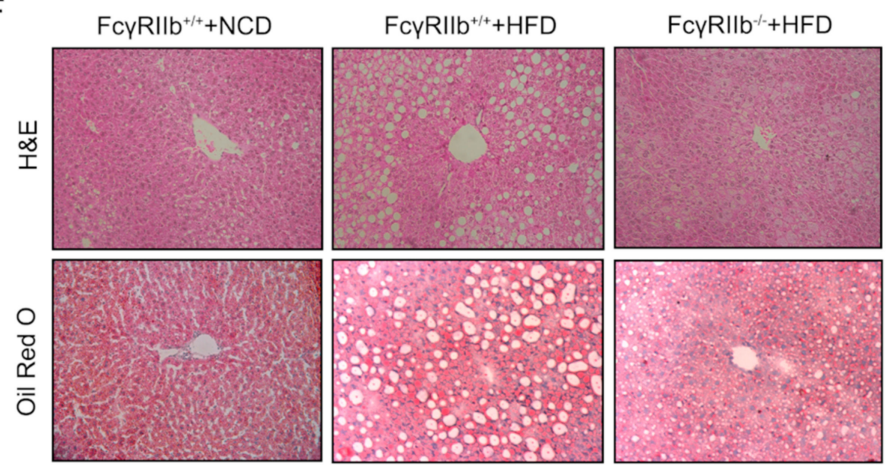

$\mathrm{H}$

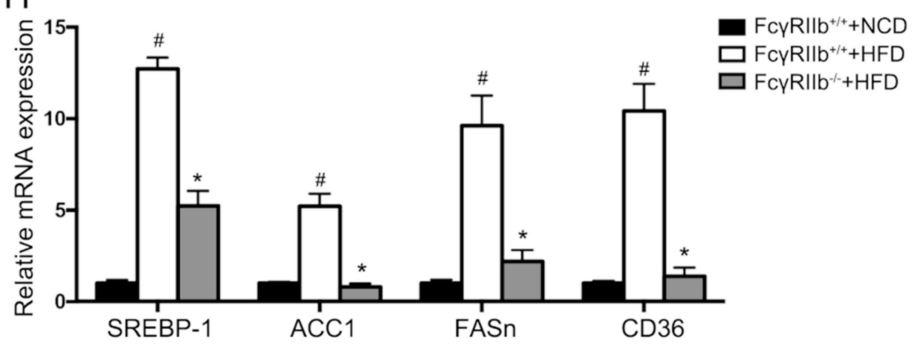

Figure 4. Effect of Fc $\gamma$ RIIb knockdown on high fat diet (HFD)-induced hepatic steatosis. (A) Body weight gain in response to normal chow diet (NCD) and HFD for Fc $\gamma \mathrm{RIIb}^{+/+}$mice, and HFD for Fc $\gamma \mathrm{RIIb}^{-/-}$mice ( $n>5$ /group). (B) Liver weight after 16 weeks of NCD and HFD feeding in Fc $\gamma \mathrm{RIIb}^{+/+}$ on NCD and HFD, Fc $\gamma \mathrm{RIIb}^{-/-}$mice on HFD ( $n>5$ /group). (C) Liver/body weight ratio of Fc $\gamma \mathrm{RIIb}^{+/+}$ on NCD and HFD, Fc $\gamma \mathrm{RIIb}^{-/}$mice on HFD for 16 weeks ( $n>5 /$ group). (D) Gross morphology changes in liver of Fc $\gamma \mathrm{RIIb}^{+/+}$mice on NCD and HFD, Fc $\gamma \mathrm{RIIb}^{-/-}$mice on HFD for 16 weeks. Arrows indicate lipid droplets in liver. (E) Liver triglyceride content in $\mathrm{Fc} \gamma \mathrm{RIIb}^{+/+}$on NCD and HFD, Fc $\gamma \mathrm{RIIb}^{-/-}$on HFD for 16 weeks ( $n>3$ /group). (F) Representative H\&E stained (top, 20×) and Oil Red O-stained (bottom,20x) images of liver sections from Fc $\gamma \mathrm{RIIb}^{+/+}$mice on NCD and HFD, Fc $\gamma \mathrm{RIIb}^{-/-}$mice on HFD for 16 weeks. (G) ALT and AST levels in the serum of Fc $\gamma \mathrm{RIIb}^{+/+}$and Fc $\gamma \mathrm{RIIb}^{-/-}$mice after 16 weeks of HFD ( $n>5$ /group). (H) qRT-PCR analysis of mRNA expression of SREBP-1, ACC1, FASn, and CD36. Total RNA was isolated from Fc $\gamma \mathrm{RIIb}^{+/+}$mice fed with NCD, and $\mathrm{Fc} \gamma \mathrm{RIIb}^{+/+}$or Fc $\gamma \mathrm{RIIb}^{-/-}$mice on HFD. The data shown are the means \pm SEM. ${ }^{\#} p<0.05$, control mice $\left(\mathrm{Fc} \gamma \mathrm{RIIb}^{+/+}\right)$of NCD vs. HFD; $* p<0.05$, HFD feeding of Fc $\gamma \mathrm{RIIb}^{+/+}$vs. Fc $\gamma \mathrm{RIIb}^{-/-}$mice. 


\subsection{FcrRIIb Decreased Hepatic Insulin Sensitivity In Vitro and In Vivo}

To investigate the role of Fc $\gamma$ RIlb in regulating hepatocyte glucose metabolism, HepG2 cells were treated with or without insulin [15], and the phosphorylation of the known mediators for insulin signaling (AKT and FOXO1) and mRNA levels of G6Pase and PEPCK (the known downstream factors of FOXO1) were examined. Insulin markedly upregulated phosphorylation of AKT and FOXO1 (lane 2 in Figure 5A), and downregulated G6Pase and PEPCK mRNA expression (bar 2 in Figure 5B). Whereas knockdown of Fc $\gamma$ RIIb with lentivirus-carried Fc $\gamma$ RIIb shRNA significantly enhanced the effect of insulin on phosphorylation of AKT and FOXO1 and the consequent mRNA expression of G6Pase and PEPCK (lane 4 in Figure 5A and bar 4 in Figure 5B), suggesting an inhibiting effect of Fc $\gamma$ RIIb on insulin-induced phosphorylation of AKT and FOXO1, and resultant decrease in G6Pase and PEPCK mRNA. The result that the knockdown of Fc $\gamma$ RIIb significantly lowered the basal level of G6Pase and PEPCK mRNA (bar 3 in Figure 5B) without markedly altering phosphorylation of AKT and FOXO1 (lane 3 in Figure 5A) suggests that Fc $\gamma$ RIIb inhibits insulin-suppressed mRNA expression of G6Pase and PEPCK through more than one mechanism.

To examine if the above finding occurs in vivo, wild type and $\mathrm{Fc}_{-} \mathrm{RIIb}^{-/-}$mice were tested in the following experiments. Compare with wild type mice, $\mathrm{Fc}_{\mathrm{R}} \mathrm{RIIb}^{-/-}$showed lower serum glucose level after $6 \mathrm{~h}$ fasting (Figure 5C), and after glucose or insulin stimulations (Glucose Tolerance Test and Insulin Tolerance Test assay) (Figure 5D,E), suggesting a better insulin sensitivity. Moreover, livers of Fc $\gamma \mathrm{RIIb}^{+/+}$and $\mathrm{Fc} \gamma \mathrm{RIIb}^{-/-}$mice were harvested to detect phosphorylation of AKT and FOXO1, and mRNA expression of G6Pase and PEPCK. In consistent with our in vitro results, FC $\gamma \mathrm{RIIb}^{-/-}$ increased phosphorylation of AKT and FOXO1 (Figure 5F,H), and decreased G6Pase, PEPCK mRNA expression (Figure 5I), indicating Fc $\gamma$ RIIb enhances insulin resistant in HFD.

A

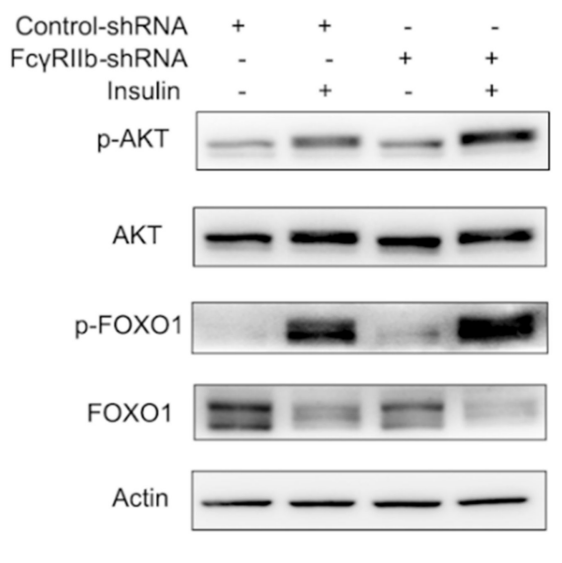

B

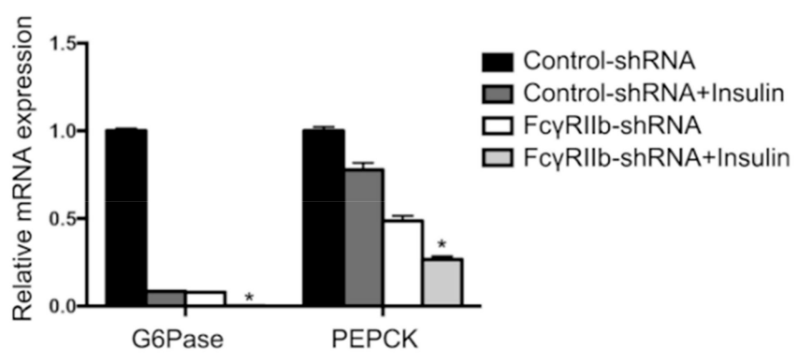

C

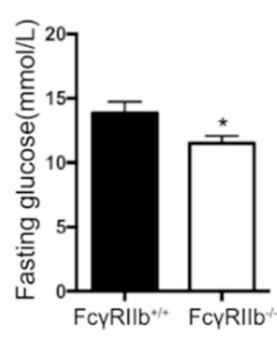

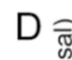

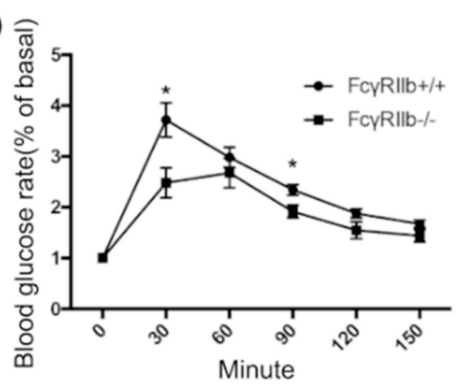

E

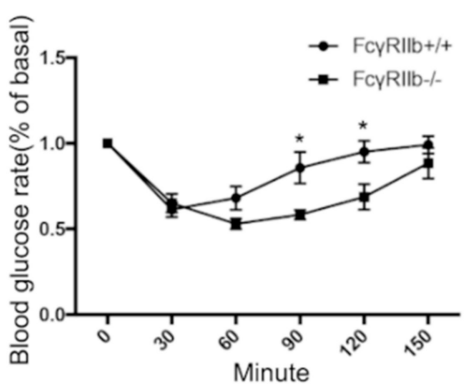

Figure 5. Cont. 

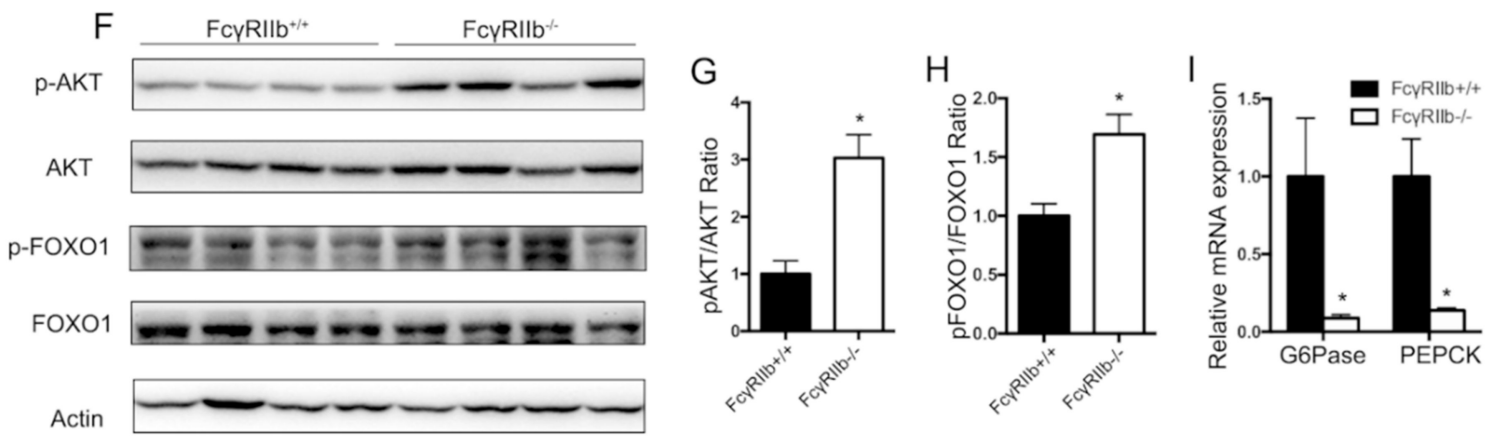

Figure 5. Effect of Fc $\gamma \mathrm{RIIb}$ on AKT phosphorylation. (A) HepG2 cells were starved for $18 \mathrm{~h}$ before insulin for $30 \mathrm{~min}$ insulin treatment. Protein and RNA were collected for western blot and qRT-PCR. Western blot analysis of insulin-induced AKT and FOXO1 phosphorylation after Fc $\gamma$ RIIb shRNA administration. HepG2 were incubated with $100 \mathrm{nmol} / \mathrm{L}$ insulin for $30 \mathrm{~min}$ after Fc $\gamma$ RIIb shRNA or control shRNA administration for $48 \mathrm{~h}$. (B) qRT-PCR of mRNA expression of the known downstream genes of FOXO1, G6Pase, and PEPCK. HepG2 cells were treated the same way as described in A. ${ }^{*} p<0.05$, between control shRNA and Fc $\gamma$ RIIb shRNA pretreatment under insulin activation. (C) Blood glucose level under fasting condition in HFD fed Fc $\gamma \mathrm{RIIb}^{+/+}$and Fc $\gamma \mathrm{RIIb}^{-/-}$mice. (D,E) GTT and ITT assay of HFD fed Fc $\gamma \mathrm{RIIb}^{+/+}$and Fc $\gamma \mathrm{RIIb}^{-/-}$mice. (F) Western blot analysis of AKT and FOXO1 phosphorylation in vivo. Fc $\gamma \mathrm{RIIb}^{+/+}$and $\mathrm{Fc} \gamma \mathrm{RIIb}{ }^{-/-}$mice were sacrificed and liver were harvested after 16 weeks of HFD. ( $n=4$ /group). (G,H) Statistical analysis of pAKT/AKT ratio and pFOXO1/FOXO1 ratio according to (F). (I) Relative mRNA expression of G6Pase or PEPCK was analyzed by qRT-PCR from liver of Fc $\gamma \mathrm{RIIb}^{+/+}$and Fc $\gamma \mathrm{RIb}^{-/-}$mice after 16 weeks HFD administration ( $n=4$ /group). The data shown are the means \pm SEM. ${ }^{*} p<0.05$.

\section{Discussion}

Fc $\gamma$ RIIb, as an IgG receptor, is commonly recognized as being widely expressed in hematopoietic cells. However, recent research demonstrates that Fc $\gamma$ RIIb is also expressed in other cells. In this report we show that both primary cultured hepatocytes and HepG2 express Fc $\gamma$ RIIb. Under OA treatment, HepG2 Fc $\gamma$ RIIb is increasingly upregulated with increasing dose and time of the treatment. Knockdown of $F c \gamma R I I b$ in HepG2 by shRNA suppresses lipid accumulation in the OA induced fatty liver cell model, and downregulates mRNA expression of genes involved in lipogenesis and lipid transport. Consistent with the in vitro results, knockout of Fc $\gamma$ RIIb inhibits the development of fatty liver in mice fed with HFD. In glucose metabolism, Fc $\gamma$ RIIb suppresses insulin-induced phosphorylation of AKT and FOXO1, while knockdown of Fc $\gamma$ RIIb ameliorates insulin-induced AKT and FOXO1 activation, and decreases G6Pase and PEPCK mRNA expression; these results are recapitulated in vivo.

Hepatocyte function includes lipid and glucose metabolism. Abnormality of lipid and glucose metabolism in hepatocytes promotes the development of fatty liver. Hepatocyte triglyceride synthesis is mainly finished by de novo lipogenesis and fatty acid uptake [3]. In this study we find that Fc $\gamma$ RIIb knockout suppresses lipid accumulation in both hepatocytes and the liver, meanwhile suppresses mRNA expression of the ACC1, FASn, CD36, MTTP and ApoB (known factors for lipid synthesis and transportation) (Figure 3), suggesting that Fc $\gamma$ RIIb enhances fatty acid uptake and esterification. Some known signaling pathways including AMP-activated protein kinase (AMPK) [16], Extracellular Regulated protein Kinases(ERK) [17], and p38 [18] are involved in the development of fatty liver. How Fc $\gamma \mathrm{RIIb}$ affects the expression of genes needs further investigation.

In our study, knockdown of Fc $\gamma$ RIIb in HepG2 exhibited a 40\% decrease in lipid accumulation (Figure 3D, lane 2 vs. lane 4). Similarly, the protective effect of Fc $\gamma \mathrm{RIIb}^{-/-}$showed nearly $70 \%$ decrease in lipid accumulation in the liver of HFD induced animal model. This 30\% difference between the in vitro and in vivo experiments may be caused by an incomplete knockdown of Fc $\gamma$ RIlb in HepG2 cells or a protective effect of Fc $\gamma$ RIIb-knockout in other cell types. Considering that the liver tissue is composed of hepatocytes, hepatic stellate cells, Kupffer cells, and sinusoidal endothelial cells, which are all reported to contribute to the development of fatty liver [19], mice with Fc $\gamma \mathrm{RIIb}^{-/-}$specific to 
hepatocytes are the best choice to verify if the mechanism identified in HepG2 exists and acts the same way in vivo. Fc $\gamma$ RIIb is an inhibitory receptor specific for IgG, known for regulating immune cells [10]. It is conceivable that $\mathrm{F} \gamma \gamma \mathrm{RIIb}^{-/-}$mice may have an inflammatory state, which may alter hepatic lipid and glucose metabolism through immune cells. It has been reported that Fc $\gamma I I b$ is expressed in macrophages [20] and B cells [21]. Kupffer cell plays an important role in fatty liver pathology though the role of B cells is unclear [19]. Kupffer cell polarization towards M1 is one of the factors that aggravate simple fatty liver into steatohepatitis [19]. Although Fc $\gamma \mathrm{Illb}$ is an inhibitory receptor, many studies showed that knockdown of Fc $\gamma \mathrm{IIb}$ ameliorates inflammation. Fc $\gamma \mathrm{Ill}$ deficiency improves atherosclerosis by enhancing macrophage M2 polarization and decreasing inflammation [20]. In our study, global Fc $\gamma \mathrm{RIIb}^{-/-}$mice showed less hepatic steatosis, which also suggests less inflammation. Nevertheless, bone marrow transplant from Fc $\gamma \mathrm{RIIb}^{+/+}$mice to $\mathrm{Fc} \gamma \mathrm{RIIb}^{-/-}$mice will provide more insight into the effect of white cells on NAFLD. Moreover, a rescue experiment in Fc $\gamma \mathrm{RIIb}^{-/-}$mice treated with an Fc $\gamma$ RIIb-carrying virus will confirm the effect of Fc $\gamma$ RIIb.

It is known that insulin stimulation activates AKT phosphorylation followed by FOXO1 phosphorylation in hepatocytes [4]. FOXO1 is a transcription factor. Phosphorylation of FOXO1 suppresses its translocation from plasma into nuclear, therefore suppresses downstream G6Pase and PEPCK expression. G6Pase and PEPCK are essential enzymes for gluconeogenesis, which cause blood glucose concentration changes in vivo [5]. Global Fc $\gamma$ RIIb deficiency improves insulin sensitivity [13]. Our study here shows that Fc $\gamma$ RIIb knockdown and knockout increase the insulin-induced phosphorylation of AKT and FOXO1 in HepG2 and the animal model (Figure 5), suggesting that Fc $\gamma$ RIIb inhibits insulin-induced activation of AKT and FOXO1, and in turn upregulates G6Pase and PEPCK in hyperlipemia in hepatocytes. This effect of Fc $\gamma$ RIIb decreases insulin sensitivity by increasing glucose generation in fatty liver. The phenomenon that Fc $\gamma$ RIIb knockdown causes more inhibition on mRNA expression of G6Pase and PEPCK without altering the basal level of AKT or FOXO1 phosphorylation (Figure $5 \mathrm{~B}$ ) suggests that $\mathrm{AKT}$ is not the only pathway mediating the inhibiting effect of Fc $\gamma$ RIIb on mRNA expression of G6Pase and PEPCK. Our results shown in Figure 5A, $F c \gamma R I I b$ shRNA treatment significantly decreased FOXO1 protein (lane 1 and lane 3), indicates that Fc $\gamma$ RIlb may regulate FOXO1 expression at mRNA or protein level, or both. By far, no reports in the literature suggest that $\mathrm{F} c \gamma \mathrm{RIIb}$ regulates mRNA transcription and stability. As for protein translation and stability, Huang et al. reported that post-translational modifications, including phosphorylation and acetylation of FOXO1, cause ubiquitination of FOXO1 in HepG2 [22]. Our current study showed that knockdown of $F c \gamma R I I b$ caused a very mild increase in Phospho-FOXO1 and a decrease in total FOXO1, suggesting a mechanism that Fc $\gamma \mathrm{RIIb}$ protects FOXO1 from ubiquitination. It is conceivable that $F c \gamma R I I b$-knockdown in HepG2 cells does not cause a marked increase in phospho-FOXO1 if phospho-FOXO1 is very unstable. As our current study is not designed to elucidate the molecular details of the effect of Fc $\gamma$ RIIb on FOXO1, we did not examine if and how Fc $\gamma$ RIIb regulates FOXO1 post-translational modification. Though Fc $\gamma$ RIIb as a tyrosine kinase receptor is able to phosphorylate its downstream factors [10], we have no data to support that FOXO1 is a direct downstream factor of Fc $\gamma \mathrm{RIIb}$. To our knowledge, it lacks information regarding direct interaction between the proteins. A successful study focusing on the interaction of these tow proteins is needed to clarify the detail of mechanism.

In summary, our study demonstrated the Fc $\gamma$ RIlb expression in hepatocyte, and the protective effect of Fc $\gamma$ RIIb deficiency on lipid accumulation and insulin resistance in hepatocytes and the liver. Blocking Fc $\gamma$ RIlb may be a useful strategy for managing NAFLD.

\section{Materials and Methods}

\subsection{Primary Hepatocyte Isolation and Culture}

Primary mouse hepatocytes were isolated from the livers of male Fc $\gamma \mathrm{RIIb}^{+/+}$and Fc $\gamma \mathrm{RIIb}^{-/-}$mice (8 weeks old in C57BL/6J background), and the protocol was as previously described [23]. Briefly, mice 
were anesthetized, and their livers were perfused with $0.5 \mathrm{mg} / \mathrm{mL}$ type II collagenase (Sigma-Aldrich, St. Louis, MO, USA), via the inferior vena cava to isolate hepatocytes. Mouse hepatocytes were cultured in RPMI-1640 containing 10\% FBS, 100 units $/ \mathrm{mL}$ penicillin, and $0.1 \mathrm{mg} / \mathrm{mL}$ streptomycin. Mouse hepatocytes were ready for experiments after 1-2 days in culture.

\subsection{Cell Culture}

HepG2 cells were purchased from ATCC (Manassas, VA, USA). Modified Eagle's medium (MEM) was bought from CORNING (10-009CVR). Insulin were from Sigma-Aldrich (I5500-10 mg). C reactive protein (CRP) was bought from APPLYGEN (Beijing, China, C2308). Oleic acid (OA) was purchased from Sigma-Aldrich, O1383.

\subsection{Animal}

Male Fc $\gamma \mathrm{RIIb}^{+/+}$and Fc $\gamma \mathrm{RIIb}^{-/-}$mice (C57BL/6J background, the Jackson Laboratory, 002848, Sacramento, CA, USA) were housed and maintained on a 12-h-light-dark cycle with a regular unrestricted diet. HF diet (Research Diets, D12108C) were fed since 6 to 8 weeks age, ad libitum, with free access to water. All animal experiments were conducted under protocols approved by the Animal Research Committee of the Institute of Laboratory Animals, Chinese Academy of Medical Sciences, and Peking Union Medical College (ACUC-A01-2014-023, 3 March 2014). Mice were weighted every week. GTT and ITT assay were performed followed standard protocol. After 16 weeks of HFD, mice were sacrificed under anesthetization, mice blood, and tissues were harvested.

\subsection{RNA Interference}

Short-hairpin RNA (shRNA)-encoding DNA sequences were synthesized by TIANYI HUIYUAN (Beijing, China) and constructed into pSIH-H1 plasmids (pSIH-H1, SI501A-1, System Biosciences, Palo Alto, CA, USA). Lentivirus were prepared by using packing plasmid psPAX2 (Addgene\#12260) and pMD2.G (Addgene\#12259). The sequence of shRNA target sequence was $5^{\prime}$-tgatgaccagaaccgtattta-3'.

\subsection{Quantitative Real-Time PCR}

Total RNA was isolated from cells or pulverized liver using TRIzol (Invitrogen, Carlsbad, CA, USA). RNA reverse transcription was using TIANGEN kit (KR103, Tiangen Biotechnology, Beijing, China). Quantitative real-time reverse-transcriptase PCR (qRT-PCR) was performed using the SYBR Green I Q-PCR kit (TransGen Biotech, Beijing, China) on a Bio-Rad IQ5 system (Bio-Rad, Hercules, CA, USA). All gene expression data were normalized to $\beta$-actin expression levels.

\subsection{Western Blot Analysis}

Proteins were extracted from frozen organ samples or cultured hepatocytes in cell lysis buffer, and $30 \mu \mathrm{g}$ of protein were loaded onto a 10\% SDS-polyacrylamide gel and separated proteins were transferred to PVDF membranes. Western blot assays were performed using antibodies specific for p-AKT, AKT, p-FOXO1, FOXO1, and Actin (Cell Signaling Technology, Danvers, MA, USA).

\subsection{Histology and Immunohistochemistry}

For H\&E and Oil Red O staining, liver tissue was embedded into OCT and frozen in liquid nitrogen and cut into $6 \mu \mathrm{m}$ sections. H\&E staining and Oil Red O staining were performed followed standard protocol.

\subsection{Triglyceride Content Assay}

Intracellular and liver triglycerides were assayed using a triglyceride assay kit (GPO-POD; Applygen Technologies Inc., Beijing, China). Intracellular and liver triglycerides were assayed using a triglyceride assay kit (GPO-POD; Applygen Technologies Inc., Beijing, China). Intracellular and liver 
triglycerides were assayed using a triglyceride assay kit (GPO-POD; Applygen Technologies Inc.). Intracellular and liver triglycerides were assayed using a triglyceride kit from APPLYGEN (Beijing, China, E1013-1015).

\subsection{Statistical Analysis}

Data are presented as means \pm SEM and were compared between or among groups by ANOVA. $p<0.05$ was considered statistically significant.

Author Contributions: Conceptualization, T.S. and J.W.; Data curation, T.S.; Formal analysis, T.S.; Funding acquisition, J.W.; Methodology, T.S., X.S., X.C., W.G., and R.C.; Project administration, J.W.; Writing original draft, T.S.

Funding: This research was funded by the Chinese Academy of Medical Sciences Innovation Fund for Medical Sciences (grant number: 2016-I2M-1-006), the National Natural Science Foundation of China (grant numbers: 81622008, 81470579, 91739107), and the Thousand Young Talents Program of China.

Acknowledgments: We thank Bin Liu for his help in manuscript preparation. We are also grateful to the anonymous reviewers and editors for their help in improving the manuscript.

Conflicts of Interest: The authors declare no conflicts of interest.

\section{Abbreviations}

$\begin{array}{ll}\text { NAFLD } & \text { Non-alcoholic fatty liver disease } \\ \text { OA } & \text { Oleic Acid } \\ \text { CRP } & \text { C Reactive Protein } \\ \text { NCD } & \text { Normal Chow Diet } \\ \text { HFD } & \text { High Fat Diet }\end{array}$

\section{References}

1. Younossi, Z.M.; Koenig, A.B.; Abdelatif, D.; Fazel, Y.; Henry, L.; Wymer, M. Global epidemiology of nonalcoholic fatty liver disease-Meta-analytic assessment of prevalence, incidence, and outcomes. Hepatology 2016, 64, 73-84. [CrossRef] [PubMed]

2. Byrne, C.D.; Targher, G. NAFLD: A multisystem disease. J. Hepatol. 2015, 62, S47-S64. [CrossRef] [PubMed]

3. Samuel, V.T.; Shulman, G.I. Nonalcoholic Fatty Liver Disease as a Nexus of Metabolic and Hepatic Diseases. Cell Metab. 2018, 27, 22-41. [CrossRef] [PubMed]

4. Samuel, V.T.; Shulman, G.I. The pathogenesis of insulin resistance: Integrating signaling pathways and substrate flux. J. Clin. Investig. 2016, 126, 12-22. [CrossRef] [PubMed]

5. Tong, X.; Zhang, D.; Charney, N.; Jin, E.; Vandommelon, K.; Stamper, K.; Gupta, N.; Saldate, J.; Yin, L. DDB1-Mediated CRY1 Degradation Promotes FOXO1-Driven Gluconeogenesis in Liver. Diabetes 2017, 66, 2571. [CrossRef] [PubMed]

6. Cohen, J.C.; Horton, J.D.; Hobbs, H.H. Human Fatty Liver Disease: Old Questions and New Insights. Science 2011, 332, 1519-1523. [CrossRef] [PubMed]

7. Kawano, Y.; Cohen, D.E. Mechanisms of hepatic triglyceride accumulation in non-alcoholic fatty liver disease. J. Gastroenterol. 2013, 48, 434-441. [CrossRef] [PubMed]

8. Rakhshandehroo, M.; Knoch, B.; Müller, M.; Kersten, S. Peroxisome proliferator-activated receptor $\alpha$ target genes. PPAR Res. 2010, 2010. [CrossRef] [PubMed]

9. Espéli, M.; Smith, K.G.; Clatworthy, M.R. Fc $\gamma$ RIIB and autoimmunity. Immunol. Rev. 2016, 269, $194-211$. [CrossRef] [PubMed]

10. Tanigaki, K.; Sundgren, N.; Khera, A.; Vongpatanasin, W.; Mineo, C; Shaul, P.W. Fc $\gamma$ Receptors and Ligands and Cardiovascular Disease. Circ. Res. 2015, 116, 368-384. [CrossRef] [PubMed]

11. Tanigaki, K.; Vongpatanasin, W.; Barrera, J.A.; Atochin, D.N.; Huang, P.L.; Bonvini, E.; Shaul, P.W.; Mineo, C. C-Reactive Protein Causes Insulin Resistance in Mice Through Fc $\gamma$ Receptor IIB-Mediated Inhibition of Skeletal Muscle Glucose Delivery. Diabetes 2013, 62, 721-731. [CrossRef] [PubMed] 
12. Tanigaki, K.; Chambliss, K.L.; Yuhanna, I.S.; Sacharidou, A.; Ahmed, M.; Atochin, D.N.; Huang, P.L.; Shaul, P.W.; Mineo, C. Endothelial Fc $\gamma$ Receptor IIB Activation Blunts Insulin Delivery to Skeletal Muscle to Cause Insulin Resistance in Mice. Diabetes 2016, 65, 1996-2005. [CrossRef] [PubMed]

13. Tanigaki, K.; Sacharidou, A.; Peng, J.; Chambliss, K.L.; Yuhanna, I.S.; Ghosh, D.; Ahmed, M.; Szalai, A.J.; Vongpatanasin, W.; Mattrey, R.F.; et al. Hyposialylated IgG activates endothelial IgG receptor Fc $\gamma$ RIIB to promote obesity-induced insulin resistance. J. Clin. Investig. 2018, 128, 309-322. [CrossRef] [PubMed]

14. Kim, J.H.; Kang, S.I.; Shin, H.S.; Yoon, S.A.; Kang, S.W.; Ko, H.C.; Kim, S.J. Sasa quelpaertensis and p-Coumaric Acid Attenuate Oleic Acid-Induced Lipid Accumulation in HepG2 Cells. J. Agric. Chem. Soc. Jpn. 2013, 77, 1595-1598.

15. Li, Z.; Zhang, H.; Liu, J.; Liang, C.P.; Li, Y.; Li, Y.; Teitelman, G.; Beyer, T.; Bui, H.H.; Peake, D.A.; et al. Reducing Plasma Membrane Sphingomyelin Increases Insulin Sensitivity. Mol. Cell. Biol. 2011, 31, 4205-4218. [CrossRef] [PubMed]

16. Smith, B.K.; Marcinko, K.; Desjardins, E.M.; Lally, J.S.; Ford, R.J.; Steinberg, G.R. Treatment of nonalcoholic fatty liver disease: Role of AMPK. Am. J. Physiol. Endocrinol. Metab. 2016, 311, E730-E740. [CrossRef] [PubMed]

17. Gu, L.Y.; Jiao, P.; Feng, B.; Li, Y.; He, Q.; Xu, H. Hepatic ERK activity plays a role in energy metabolism. Mol. Cell. Endocrinol. 2013, 375, 157-166. [CrossRef] [PubMed]

18. Gu, L.Y.; Qiu, L.W.; Chen, X.F.; Lü, L.; Mei, Z.C. Oleic Acid-Induced Hepatic Steatosis is Coupled with Downregulation of Aquaporin 3 and Upregulation of Aquaporin 9 via Activation of p38 Signaling. Horm. Metab. Res. 2014, 47, 259-264. [CrossRef] [PubMed]

19. Bieghs, V.; Trautwein, C. The innate immune response during liver inflammation and metabolic disease. Trend. Immunol. 2013, 34, 446-452. [CrossRef]

20. Mendez-Fernandez, Y.V.; Stevenson, B.G.; Diehl, C.J.; Braun, N.A.; Wade, N.S.; Covarrubias, R.; van Leuven, S.; Witztum, J.L.; Major, A.S. The inhibitory Fc $\gamma$ RIlb modulates the inflammatory response and influences atherosclerosis in male apoE ${ }^{-/-}$mice. Atherosclerosis 2011, 214, 73-80. [CrossRef] [PubMed]

21. Zou, X.; Cutler, A.J.; Brownlie, R.J.; Fairfax, K.; Lawlor, K.E.; Severinson, E.; Walker, E.U.; Manz, R.A.; Tarlinton, D.M.; Smith, K.G.C. Fc $\gamma$ RIIb controls bone marrow plasma cell persistence and apoptosis. Nat. Immunol. 2007, 8, 419-429.

22. Huang, H.; Tindall, D.J. Regulation of FOXO protein stability via ubiquitination and proteasome degradation. Biochim. Biophys. Acta 2011, 1813, 1961-1964. [CrossRef] [PubMed]

23. Zhang, H.; Chen, Q.; Yang, M.; Zhu, B.; Cui, Y.; Xue, Y.; Gong, N.; Cui, A.; Wang, M.; Shen, L.; et al. Mouse KLF11 regulates hepatic lipid metabolism. J. Hepatol. 2013, 58, 763-770. [CrossRef] [PubMed] 